\title{
Loss of Bmyc results in increased apoptosis associated with upregulation of Myc expression in juvenile murine testis
}

\author{
Heikki T Turunen ${ }^{1,2}$, Petra Sipilä ${ }^{1,3,4}$, Leena Strauss ${ }^{1,3}$, Ida Björkgren ${ }^{1,2}$, Ilpo Huhtaniemi ${ }^{1,5}$ \\ and Matti Poutanen ${ }^{1,3}$ \\ ${ }^{1}$ Department of Physiology, Institute of Biomedicine, University of Turku, Kiinamyllynkatu 10, FIN-20520 Turku, \\ Finland, ${ }^{2}$ Turku Graduate School of Biomedical Sciences, Kiinamyllynkatu 13, FIN-20520 Turku, Finland, \\ ${ }^{3}$ Turku Center for Disease Modeling, Kiinamyllynkatu 10, FIN-20520 Turku, Finland, ${ }^{4}$ Institute of Biomedicine, Viikki \\ Biocenter, University of Helsinki, 00014 Helsinki, Finland and ${ }^{5}$ Imperial College London, Institute of Reproductive \\ and Developmental Biology, Hammersmith Campus, London W12 ONN, UK
}

Correspondence should be addressed to M Poutanen at Department of Physiology and Turku Center for Disease Modeling, Institute of Biomedicine, University of Turku; Email: matti.poutanen@utu.fi

\begin{abstract}
Bmyc is a member of the Myc family of transcriptional regulators in the mouse and the rat. It is predominantly expressed in hormonally controlled tissues, with highest level of expression in the epididymis. The BMYC protein has been shown to function as a transcription factor in vitro and to inhibit MYC. To study the significance of BMYC in vivo, a Bmyc knockout (KO) mouse model was generated by homologous recombination. The $\mathrm{KO}$ mice were viable and fertile and did not display gross morphological or histological changes compared to the WT mice. However, the testes and the epididymides of the KO mice were smaller than those of the WT mice.

Correspondingly, a tendency for a lower sperm concentration in the cauda epididymides of the KO mice was detected. The testosterone produced/testis was significantly reduced, and accordingly, the LH levels were increased in the KO mice. Also, the expression levels of Myc and several of its target genes were elevated in the testes of prepubertal KO mice, whereas no differences in gene expression levels were detected in adult mice. Associated with the increased Myc expression, more apoptotic spermatogenic cells were detected in the seminiferous tubules of the KO mice. In conclusion, our data suggest that Bmyc is a regulator of Myc in vivo and that overexpression of Myc in the developing testis leads to increased apoptosis of spermatogenic cells.

Reproduction (2012) 144 495-503
\end{abstract}

\section{Introduction}

The epididymis is a mammalian organ responsible for post-testicular sperm maturation and storage of mature sperm. Recent advances in high-throughput genome approaches have led to an intensive search of genes coding for proteins involved in epididymal sperm maturation processes (Sipilä et al. 2009). As many of these genes are expressed exclusively in the epididymis, the identification of transcription factors regulating their expression is also of interest, although still only superficially known. Bmyc codes for a transcription factor abundantly expressed in the epithelial cells of the proximal caput epididymis, and the expression diminishes dramatically toward the cauda (Cornwall et al. 2001).

Bmyc is a relatively poorly known member of the Myc family of transcriptional regulators. It was originally identified in the rat and named after its high expression in the brain (Ingvarsson et al. 1988). Subsequently, an even higher expression was reported in the mouse epididymis where its expression is regulated by androgens (Gregory et al. 2000, Cornwall et al. 2001). Lower levels of expression have been detected in several other hormonally controlled tissues, including the adrenal, pituitary, prostate, ovary, and uterus, and the protein has also been detected in the hypothalamus and testis (Gregory et al. 2000). In addition, Bmyc is the predominant Myc family gene expressed during mouse preimplantation development (Domashenko et al. 1997).

The BMYC protein has high homology with the $\mathrm{N}$-terminal domain of MYC and a unique 14 amino acid C-terminal domain that is not found in other MYC family proteins (Ingvarsson et al. 1988, Asker et al. 1995). BMYC lacks a nuclear localization signal and a basic helix-loop-helix leucine zipper (b-HLH-LZ) DNA binding domain typical for other MYC proteins that is also required for dimerization with MAX. However, it contains a transcriptional activation domain (TAD), localizes mainly in the nucleus, and has been shown in vitro to initiate transcription, to inhibit neoplastic 
transformation, and to inhibit transcriptional activation regulated by MYC (Resar et al. 1993, Asker et al. 1995). BMYC has also been shown to inhibit cellular proliferation (Gregory et al. 2000).

The MYC-MAX complexes preferably recognize and bind to E-box sequences (CACGTG) in the genome (Blackwood \& Eisenman 1991, Henriksson \& Luscher 1996). As BMYC lacks the domain necessary for dimerization with MAX, it must mediate its gene expression regulatory actions via other mechanisms. Due to their high degree of homology, it has been suggested that transcriptional regulation may be partly mediated by competition for the same binding partners with MYC (Resar et al. 1993). BMYC has also been shown to be phosphorylated in the TAD at sites conserved with MYC, which further implies interactions with the same partners (Gregory et al. 2000). In addition to b-HLH-LZ-mediated binding, two regions responsible for other interactions, namely MYC boxes I and II (MBI and $\mathrm{MBII}$ ), are well conserved amongs all MYC proteins (Henriksson \& Luscher 1996). MBII, in particular, has been identified as a critical domain in regulating virtually all biological activities of MYC (Cole \& McMahon 1999). MYC interacting proteins PFDN5 (MM1) and TBP have also been shown to bind to MBI and MBII domains of BMYC (Burton et al. 2006).

So far no functional data for BMYC in the epididymis have been available. However, the expression of Bmyc was downregulated 500-fold and Myc was correspondingly upregulated 2.6-fold in caput epididymis of a mouse model for epididymal tumorigenesis (Sipilä et al. 2004). Given the rarity of epididymal tumors (Ganem et al. 1998), and the ability of BMYC to inhibit MYC-mediated neoplastic transformation (Resar et al. 1993), this has led to hypothesis that BMYC could act as a tumor suppressor in the epididymis (Sipilä et al. 2004). To address the role of Bmyc in epididymal tumorigenesis and in the development and function of the epididymis, in this study, we have created and characterized the Bmyc knockout (KO) mice.

\section{Results}

\section{Generation of the Bmyc KO mice}

Coding sequence of Bmyc was interrupted by targeted insertion of neomycin resistance gene (neor) cassette (Fig. 1A). Correct targeting was confirmed by Southern hybridization and PCR, and loss of Bmyc mRNA from the caput epididymis was verified by RT-PCR (Fig. 1B, C and D). Male and female $\mathrm{HE}$ and $\mathrm{KO}$ mice were fertile and obtained litters comparable in size to WT mice $(6.6 \pm 2.6,7.7 \pm 2.9$, and $7.7 \pm 1.8$ pups/l for $\mathrm{WT}, \mathrm{HE}$, and $\mathrm{KO}$ breedings respectively with 14,32 , and $20 \mathrm{I}$ studied respectively). However, Mendelian distribution of genotypes was not observed from $\mathrm{HE} \times \mathrm{HE}$ breedings; out of 260 genotyped pups, only $43 \mathrm{KO}$ mice were observed, as opposed to the expected $65\left(\chi^{2}\right.$ test, $\left.P<0.01\right)$, while no significant differences in gender distributions were detected. Otherwise the KO mice appeared healthy and normal. At the ages of 2 and 3 weeks, the $\mathrm{KO}$ males weighed significantly less than WT males $(6.7 \pm 1.2$ and $8.4 \pm 1.4 \mathrm{~g}$ at 2 weeks, $n=8$ and 6 respectively and $9.4 \pm 1.3$ and $11.2 \pm 1.1 \mathrm{~g}$ at 3 weeks, $n=19$ and 7 respectively). A trend of decreased body weight was also detected in male $\mathrm{KO}$ mice over 4 weeks of age, but the weight difference was not statistically significant.

\section{Changes in male reproductive organs in Bmyc $\mathrm{KO}$ mice}

The weight of the testes and the epididymides of the $\mathrm{KO}$ as well as their weights relative to whole body weights were smaller than those of WT mice in all age groups studied (Fig. 2A, B and C). As the weights of the testis and the epididymis are mainly constituted of the amounts of spermatozoa, sperm production was estimated by comparing caudal sperm concentrations in the 3-month-old WT and KO mice. The sperm concentrations measured for the $\mathrm{KO}$ and WT mice were $6.1 \pm 2.1 \times 10^{6} / \mathrm{ml}$ and $7.4 \pm 4.5 \times 10^{6} / \mathrm{ml}(n=6$ and 9$)$ respectively. This is well in line with the detected decreases in adult testis (17\%) and epididymis (15\%)
A

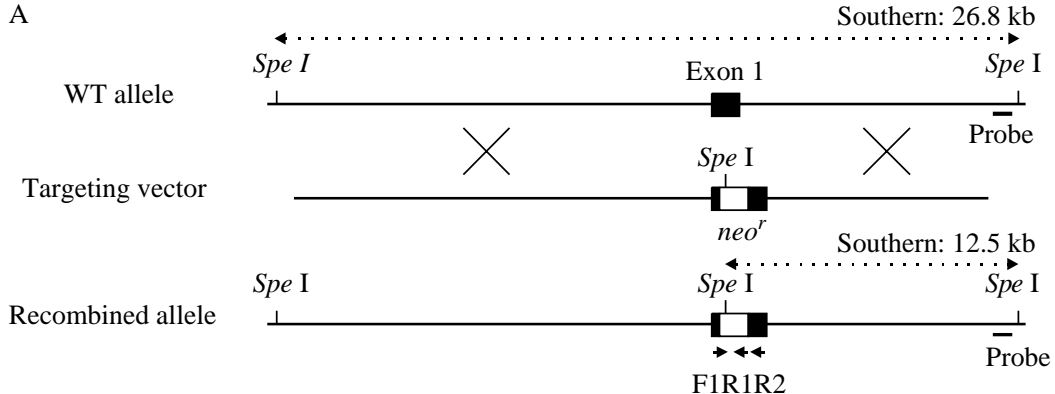

B

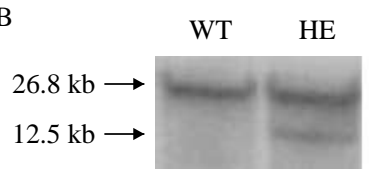

Reproduction (2012) 144 495-503

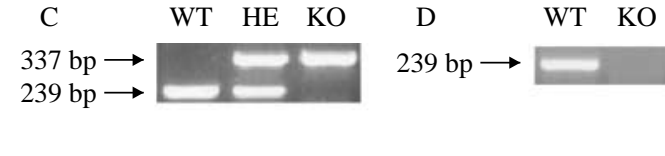

Figure 1 Generation of the Bmyc KO mice. (A) Vector used for targeting and the structure of the recombined allele after successful targeting. (B) Southern blot of correctly targeted (HE) and WT control clones. Spel digestion sites, predicted fragment lengths, and probe location are marked in panel A. (C) Genotyping of KO mice. Primers used for genotyping (F1, R1, and R2) are marked in panel A. (D) Bmyc RT-PCR of total RNA extracted from the initial segment (IS) epididymis of WT and KO mice. F1 and R2 primers (panel A)

were used. 

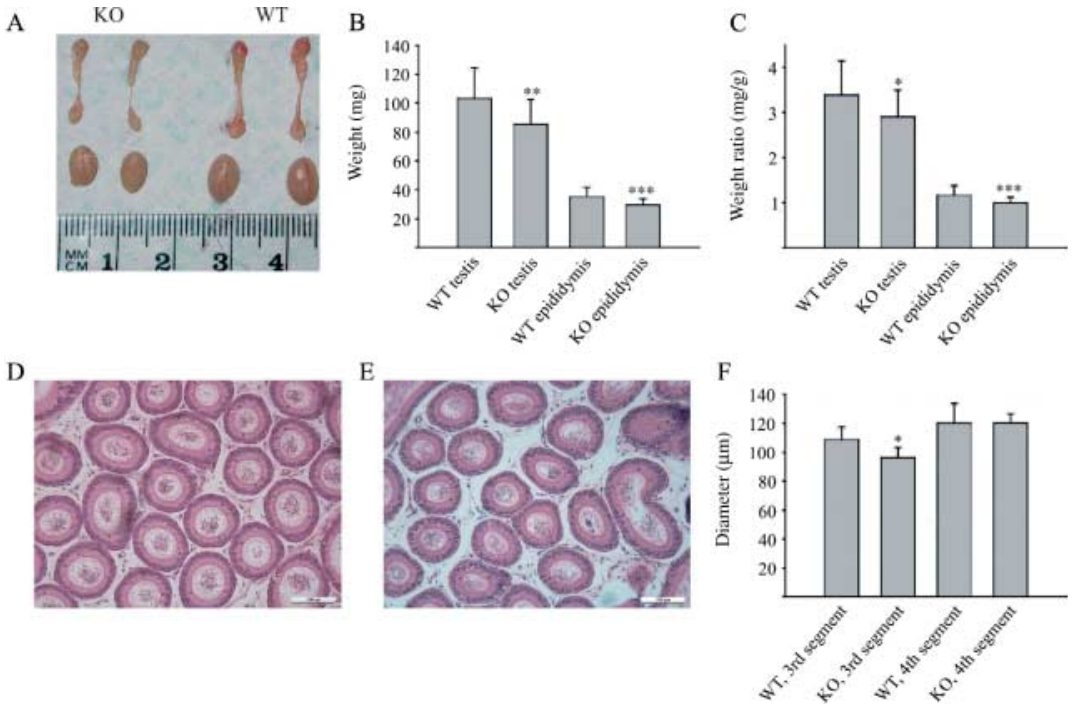

Figure 2 Male reproductive organ sizes and epididymal histology. (A) Testes and epididymides of 3-month-old WT and KO mice. (B) Testis and epididymis weights of 3-month-old WT and $\mathrm{KO}$ mice. $n=20-34, * P<0.05,{ }^{*} P<0.01$, $* * * P<0.001$. (C) Testis and epididymis weights relative to body weights of 3 -month-old WT and KO mice. $n=20-34,{ }^{*} P<0.05,{ }^{* * *} P<0.001$.

(D) Histological section of 3-month-old WT caput epididymis. Scale bar $=100 \mu \mathrm{m}$. (E) Histological section of 3-month-old KO caput epididymis. Scale $\mathrm{bar}=100 \mu \mathrm{m}$. (F) Mean diameters of the epididymal duct in segments 3 and 4 of caput epididymis in 3-month-old WT and KO mice. $n=5, * P<0.05$. S.D. are shown as error bars in all graphs. weights in the $\mathrm{KO}$ males. However, in contrast to that of the epididymis and testis weights, the difference in sperm concentrations did not reach the level of statistical significance. The KO spermatozoa appeared normal in morphology and in motility based on visual assessments. Histological analyses neither revealed gross abnormalities in the KO epididymides (Fig. 2D and E). However, in two out of the $37 \mathrm{KO}$ mice studied, atrophied testicular seminiferous tubules devoid of spermatogenic cells were observed. Furthermore, the diameter of the epididymal duct was significantly smaller in segment 3 of the $\mathrm{KO}$ caput epididymis compared with the WT mice $(P<0.05 ;$ Fig. 2F). However, no difference in diameters was detected in segment 4 (Fig. 2F). According to the smaller sizes of the male reproductive organs, a lower testosterone production per testis was measured in adult
KO compared with the WT mice $(P<0.05$; Fig. $3 \mathrm{~A})$, and it was accompanied with a significant increase in serum LH levels in 3-month-old mice ( $P<0.01$; Fig. 3C). In contrast to the reduced testosterone production, the serum level of testosterone was not significantly altered, and no difference was detected in FSH levels between the $\mathrm{KO}$ and WT mice (Fig. 3B and D).

\section{Changes in gene expression levels in Bmyc $\mathrm{KO}$ mice}

We compared the expression levels of $M y c$ and its direct downstream target genes Brca1, Cdk11b(Cdc2l1), Cdk4, and Mina between WT and KO mice in the testes and the epididymides at different ages. No changes in expression levels in the epididymis were detected, whereas $M y_{c}$ and $C d k 4$ were statistically significantly upregulated in

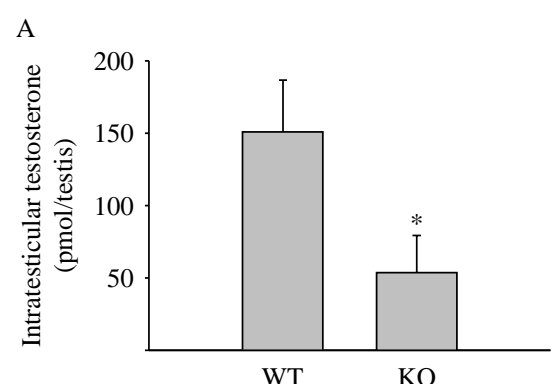

C

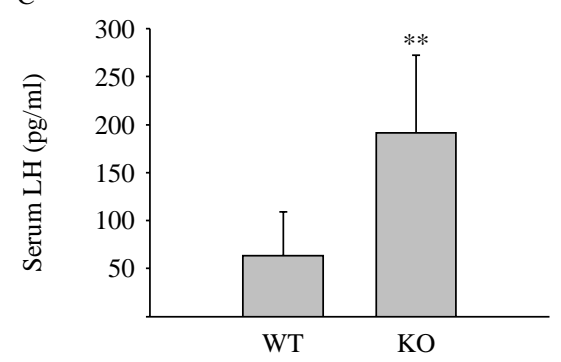

www.reproduction-online.org

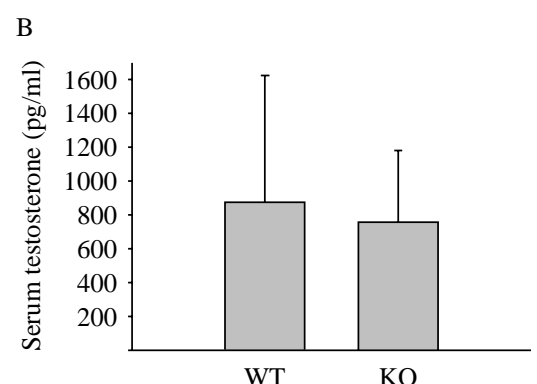

D

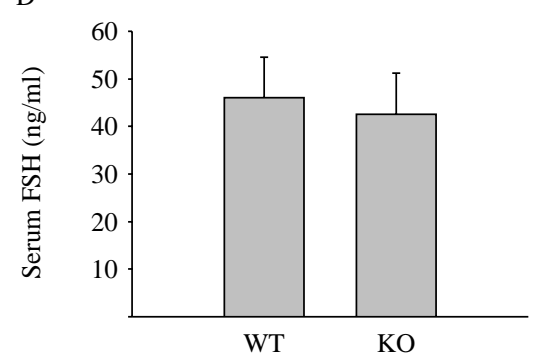

Figure 3 Serum and intratesticular hormone levels. (A) Intratesticular testosterone levels of 3-monthold WT and KO mice. $n=8$ and 11 respectively, $* P<0.05$. (B) Serum testosterone levels of 3-month-old WT and KO mice. $n=5$ and 11 respectively. (C) Serum $\mathrm{LH}$ levels of 3-month-old WT and KO mice. $n=5$ and 11 respectively, $* * P<0.01$. (D) Serum FSH levels of 3-month-old WT and KO mice. $n=5$ and 11 respectively. S.D. are shown as error bars in all graphs. 
the testes of 3 -week-old KO mice $(P<0.001$ and $P<0.01$ respectively; Fig. $4 \mathrm{~A}$ and $\mathrm{B}$ ) while statistically significant increases were not identified for Cdk11b, Brca1, and Mina (Fig. 4C, D and E).

\section{Apoptosis and cell proliferation in the testes of the Bmyc $K O$ mice}

The increased Myc expression prompted us to compare rates of proliferation and apoptosis in the testes between 3-week-old WT and KO mice. No changes in the amounts of proliferating cells as determined by $\mathrm{Ki}-67$ staining were detected (Fig. 5A, B, C and D). However, TUNEL staining revealed significantly more apoptotic cells in the seminiferous tubules of the $\mathrm{KO}$ mice than in the WT mice, whereas the percentage of tubules with no apoptotic cells was significantly smaller in the $\mathrm{KO}$ than in the WT mice (Fig. 6A, B and C). At worst, the increased apoptosis resulted in seminiferous tubules completely devoid of spermatogenic cells (Fig. 6D).

\section{Induction of cell proliferation in the epididymides of the Bmyc KO mice}

We also studied the putative role of BMYC in epididymal tumorigenesis by crossing the Bmyc KO mice with Gpx5-Tag2 transgenic mice (Sipilä et al. 2004). In the mouse line with high SV40 Tag expression levels Gpx5-Tag1, $100 \%$ of mice develop tumors in the epididymis, whereas in the other mouse line with low SV40 Tag expression, GPX5-Tag2 mice, the T-antigen expression leads to hyperplastic epithelial changes and infertility, but no tumors are formed. Thus, the Bmyc KO mice were crossbred with GPX5-Tag2 mice expressing low levels of SV40 Tag in the epididymis to study whether the loss of Bmyc expression makes epididymis more susceptible to tumorigenesis. The data revealed that the loss of Bmyc did not induce tumorigenesis in the double transgenic mice, while the epididymal histology of the Bmyc KO/Gpx5-Tag2 mice was not distinguishable from the Gpx5-Tag2 mice used as a control.
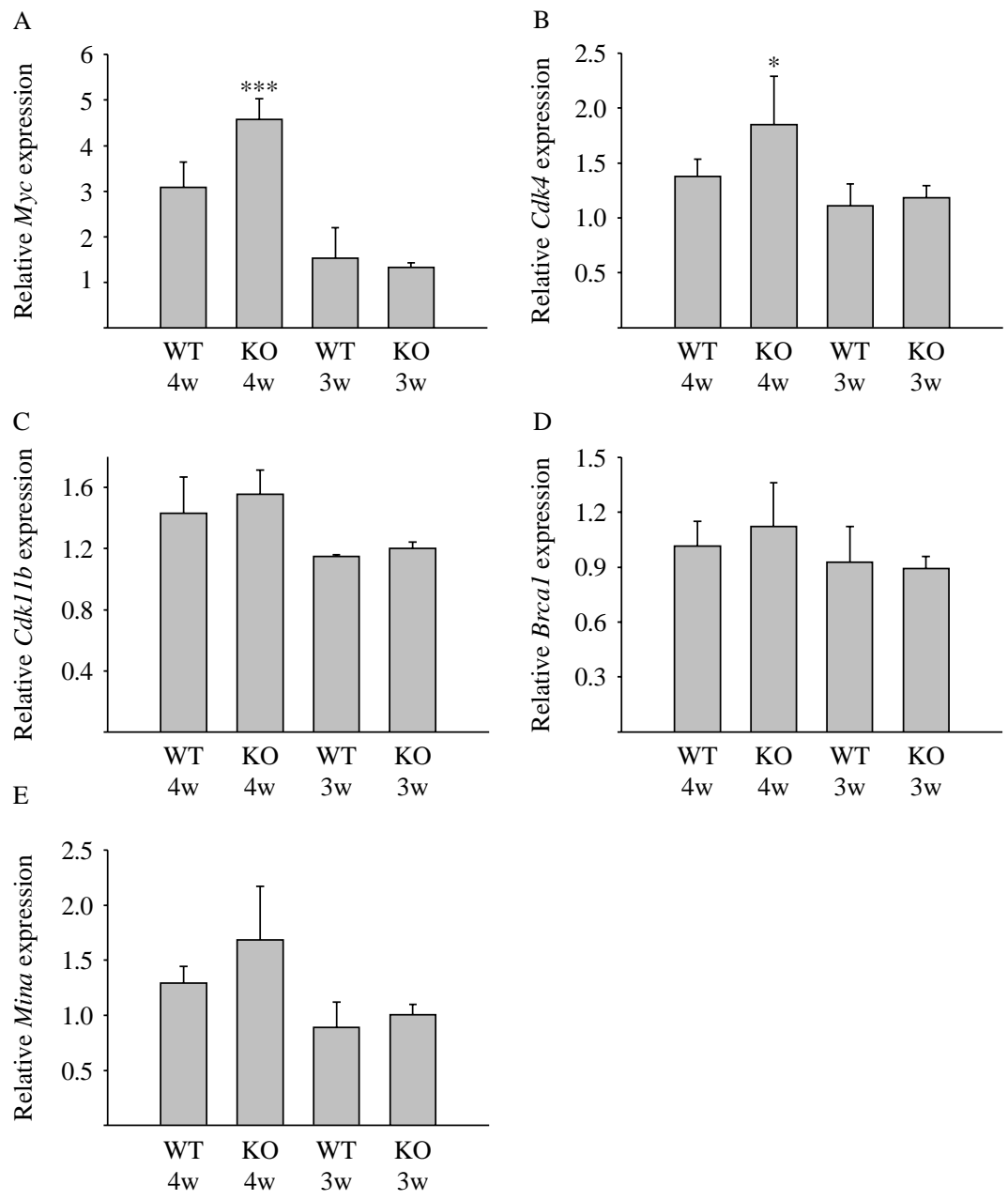

Figure 4 Gene expression in the testis. (A) Expression of $M y c$ relative to Actb expression in 4-week-old and 3 -month-old WT and KO mice. $n=6$ and 3 for 4 weeks and 3 months respectively, ${ }^{* * *} P<0.001$. (B) Expression of $C d k 4$ relative to Actb expression in 4-week-old and 3-month-old WT and KO mice. $n=6$ and 3 for 4 weeks and 3 months respectively, ${ }^{*} P<0.05$. (C) Expression of $C d k 11 b$ relative to Actb expression in 4-week-old and 3-month-old WT and KO mice. $n=6$ and 3 for 4 weeks and 3 months respectively. (D) Expression of Brca1 relative to Actb expression in 4-week-old and 3-month-old WT and KO mice. $n=6$ and 3 for 4 weeks and 3 months respectively. (E) Expression of Mina relative to Actb expression in 4-week-old and 3-month-old WT and KO mice. $n=6$ and 3 for 4 weeks and 3 months respectively. S.D. are shown as error bars in all graphs. 

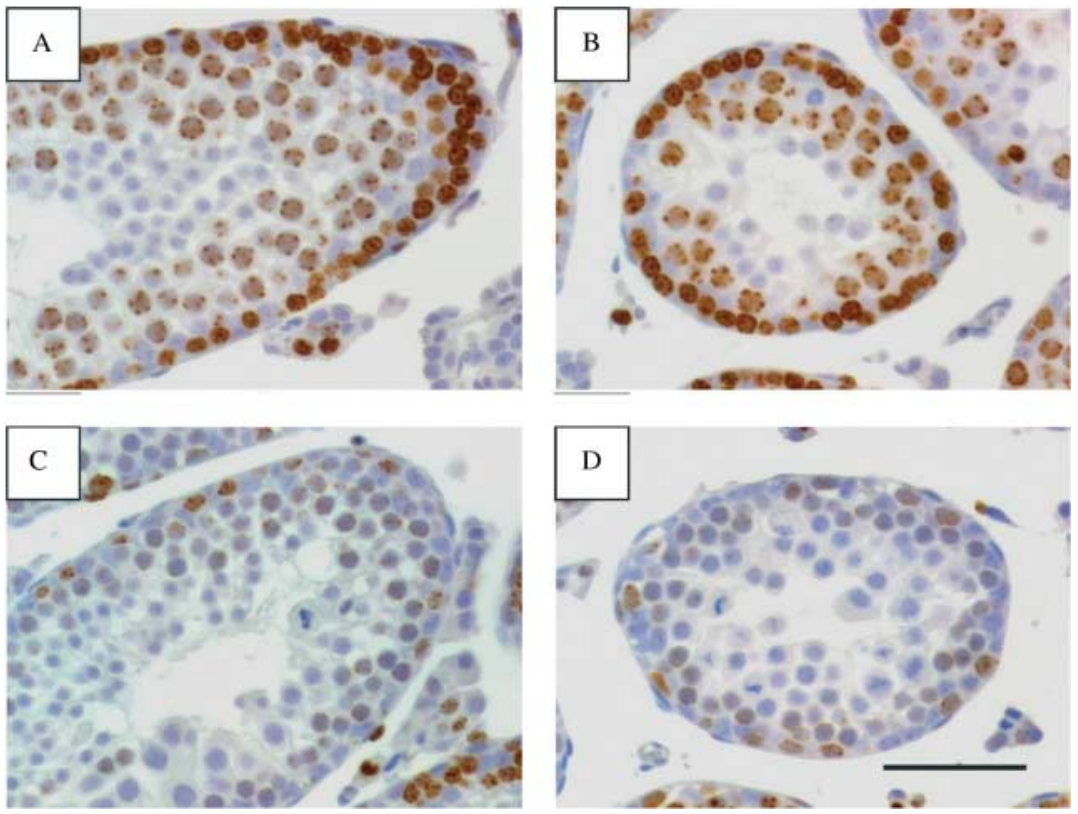

Figure 5 Proliferation in the testis. Ki67 immunohistochemical staining of a 3-week-old (A) WT mouse and (B) KO mouse at stages $\mathrm{V}-\mathrm{VI}$, (C) WT mouse, and (D) KO mouse at stage XII. No changes in the amounts of proliferating cells were detected.

\section{Discussion}

As an inhibitor of MYC, BMYC is potentially an important regulator of several cellular processes ranging from proliferation and growth to apoptosis (reviewed in Hoffman \& Liebermann (2008) and Albihn et al. (2010)). The high expression of Bmyc in the blastocyst suggests involvement in early embryonic development, whereas in the adult the highest expression levels have been detected in hormonally controlled organs. Along with the ability to inhibit MYC, expression of Bmyc in the brain and the epididymis, organs with low incidence of primary tumors (reviewed in Ganem et al. (1998) and Salacz et al. (2011)), suggests that BMYC may be a tumor suppressor. Furthermore, Bmyc expression was lost in a mouse model of epididymal tumorigenesis, and this was associated with an increased Myc expression and severe epididymal hyperplasia (Sipilä et al. 2004). Although potentially an important transcriptional regulator, the
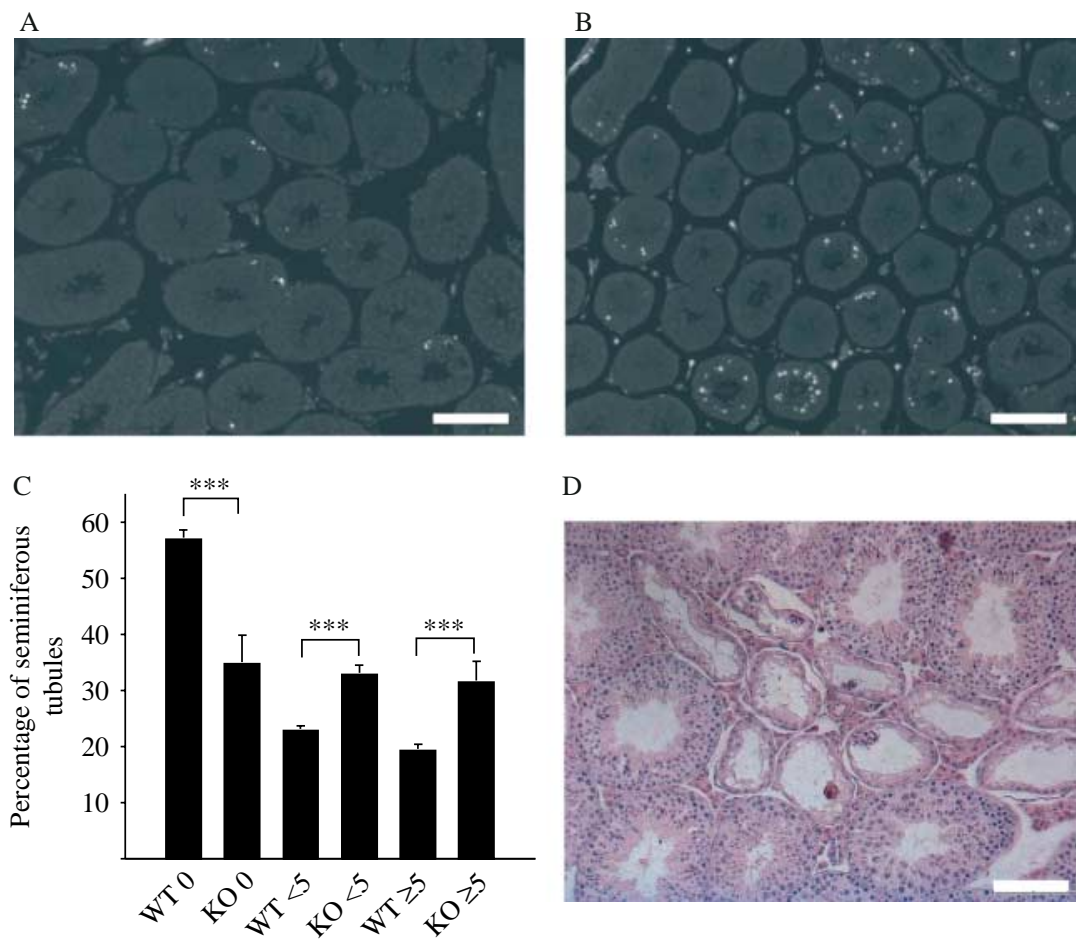

D

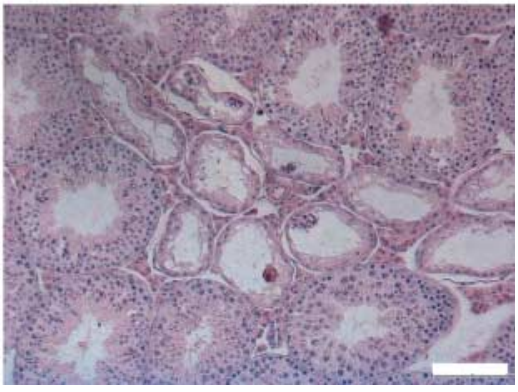

Figure 6 Apoptosis in the testis. (A) TUNEL staining of the testis of a 3-week-old WT mouse. (B) TUNEL staining of the testis of a 3-week-old $\mathrm{KO}$ mouse. (C) Percentages of seminiferous tubules with 0 , $<5$, and 5 or more TUNEL-positive cells in 3-week-old WT and KO mice. $n=4$ (WT) and $3(\mathrm{KO}),{ }^{* * *} P<0.001$. S.D. are shown as error bars. (D) Hematoxylin and eosin staining of 3-month-old KO testis showing a cluster of atrophied seminiferous tubules. 
role of BMYC has mainly been studied in vitro. To further increase our knowledge of its significance in vivo, we have generated and characterized a Bmyc KO mouse model.

Despite its high expression in early embryonic development and the lethality caused by the loss of Myc or Mycn (Nmyc) (Davis et al. 1993, Domashenko et al. 1997, Hurlin 2005), homozygous Bmyc KO mice were obtained. Although the $\mathrm{KO}$ mice proved mainly healthy and fertile, HEXHE breedings produced less homozygotes than expected by Mendelian distribution. Along with the detected smaller body weight of juvenile $\mathrm{KO}$ mice, this suggests a slight delay in the development of the KO embryos compared with the WT and KO embryos, leading to the detected increased mortality of the $\mathrm{KO}$ embryos in an environment shared with the normally developing WT and KO embryos. Although occasional embryo resorption sites were detected in uteri after $\mathrm{HE} \times \mathrm{HE}$ breedings, the underlying reason behind the decrease in the amount of $\mathrm{KO}$ pups from $\mathrm{HE} \times \mathrm{HE}$ breedings remains unknown.

The ability of BMYC to regulate gene transcription and its high expression in the epididymis suggested a role for the protein in the development or function of the epididymis. Although the KO mice were fertile, the epididymides were small in size and weighed less than the WT epididymides with a tendency for a lower sperm concentration in the cauda epididymis. While not statistically significant, the decrease in mean sperm concentrations corresponded with the decreased epididymal weights. Interestingly, the $\mathrm{KO}$ testes were also small, while testicular histology was normal in most of the $\mathrm{KO}$ animals. High serum $\mathrm{LH}$ levels indicate a hypogonal phenotype of the $\mathrm{KO}$ mice with a reduced capacity of the smaller testes to respond to pituitary $\mathrm{LH}$ stimulus. The serum testosterone concentration was, however, normalized by the increased LH stimulus, and the reduced epididymal size is, thus, likely to be caused mainly due to reduced luminal testosterone concentration.

Although the expression of Bmyc in the testis has not been previously reported, we set out to characterize Bmyc expression in the testes of juvenile mice, and, indeed, expression levels higher than in adults were detected. As BMYC has been shown to inhibit MYC in vitro, we estimated MYC activity in the testes of the Bmyc KO mice by measuring expression levels of $M y c$ and its known target genes Brca1, Cdk11b,Cdk4 and Mina (Menssen \& Hermeking 2002, Tsuneoka et al. 2006). Interestingly, the expression of Myc itself was significantly upregulated in the testes of 3-week-old $\mathrm{KO}$ mice, and similarly, upregulation of one of the Myc target genes, $C d k 4$, was detected. The upregulation of Myc expression suggests that either BMYC directly regulates the transcription of $M y c$ or the increased MYC activity due to the loss of BMYC leads to an increase in its expression by an autoregulatory pathway.
Although Myc expression and function in the testis have not been extensively studied, transgenic mouse (Suzuki et al. 1996) and rat (Kodaira et al. 1996) models overexpressing Myc in testicular cells have been generated. In both models, several founder lines displayed excessive apoptosis of spermatogenic cells and atrophy of the seminiferous tubules but also transgenic founder lines with morphologically normal testes were detected. However, the transgene expression levels of the different lines were not measured. A similar variable phenotype was detected in the $\mathrm{KO}$ mouse model of $p 19^{\operatorname{lnk} 4 d}$, an inhibitor of CDK4. Out of seven p19 ${ }^{\operatorname{lnk} 4 d}$ KO mice studied, two displayed atrophied seminiferous tubules (Buchold et al. 2007). Unfortunately, the authors did not measure $C d k 4$ expression levels. Thus, the required increase in Myc and/or Cdk4 expression causing apoptosis in spermatogenic cells is not known. In Bmyc KO mice, we detected 1.5- and 1.3-fold increases in the testicular expression levels of Myc and Cdk4 respectively. However, even these minor and transient changes in Myc and Cdk4 expression levels resulted into a clear increase in apoptosis in the testis and to the presence of seminiferous tubules devoid of spermatogenic cells with low incidence.

In conclusion, our data show that the loss of Bmyc causes upregulation of $M y c$ during testicular development, and leads to increased apoptosis of spermatogenic cells in the seminiferous tubules, which results in smaller size of the testes and the epididymides. The smaller testes produce less testosterone, which is compensated by the increased LH production. These observations indicate that BMYC functions as a transcriptional regulator in vivo. The lower body weights of the juvenile Bmyc $\mathrm{KO}$ mice compared with WT mice indicate a role for BMYC during development in proliferating tissues. Also, as a regulator of $M y c$, the effect of BMYC during tumorigenesis is of interest and may warrant further studies with tumorigenic mouse models.

\section{Materials and Methods}

\section{Generation of the Bmyc KO mice}

A genomic BAC clone (RP22-380G15) containing the Bmyc locus was purchased from Children's Hospital Oakland Research Institute (Oakland, CA, USA). Cloning of the targeting construct was performed by the Red/ET recombination technology (Zhang et al. 1998, 2000, Muyrers et al. 1999). The coding sequence of Bmyc was interrupted by inserting a $n e o^{r}$ in the exon 1 of the Bmyc gene, and the modified locus, with $15.3 \mathrm{~kb}$ upstream and $10.4 \mathrm{~kb}$ downstream homology arms (Fig. 1A), was targeted to AB2.2 embryonic stem (ES) cells (Lexicon Genetics, Houston, TX, USA). The cells were cultured in the presence of geneticin (G-418, $350 \mu \mathrm{g} / \mathrm{ml}$, Gibco, Invitrogen) for 8 days, and the antibiotic resistant colonies were picked, expanded, and stored frozen at $-70{ }^{\circ} \mathrm{C}$.

Correct targeting of the construct was assessed with negative backbone PCR (McDermott et al. 2004) and Southern 
hybridization. The negative backbone PCR was performed by amplifying a $576 \mathrm{bp}$ fragment of the vector backbone with primers neoF and neoR (Table 1) and a native DyNAzyme I DNA Polymerase (FinnZymes Diagnostics, Espoo, Finland). Based on the results, more than $90 \%$ of the clones were excluded. Correct targeting in the remaining ES cell colonies was confirmed with Southern hybridization analyses. Genomic DNA $(5-15 \mu \mathrm{g})$ was digested with Spel (Promega), sizefractioned in $0.7 \%$ SeaKem Gold Agarose (Cambrex, East Rutherford, NJ, USA) gel, and transferred to Hybond XL (Amersham Biosciences) membranes. The membranes were hybridized overnight at $42{ }^{\circ} \mathrm{C}$ in ULTRAhyb buffer (Ambion, Austin, TX, USA) according to the manufacturer's protocol. A 600 bp-long PCR fraction 3' end of the Bmyc locus, labeled with $\left[\alpha^{-32} \mathrm{P}\right] \mathrm{dCTP}$ (Prime-a-Gene Labelling System, Promega), was used as a probe. The signal was detected with Fuji Super RX film (Fujifilm, Tokyo, Japan). The expected sizes for the WT and targeted alleles were 26.8 and $12.5 \mathrm{~kb}$ respectively.

Chimeric mice were generated by injecting a correctly targeted ES cell clone into $\mathrm{C} 57 \mathrm{BL} / 6 \mathrm{~N}$ blastocysts. Chimeric males were bred with $\mathrm{C} 57 \mathrm{BL} / 6 \mathrm{~N}$ females to obtain heterozygous (HE) mice, which were further bred to obtain Bmycdeficient homozygous $\mathrm{KO}$ mice (Bmyc KO). The mice were genotyped by PCR using primers $\mathrm{F} 1, \mathrm{R} 1$, and $\mathrm{R} 2$ in the same reaction (Table 1). The $\mathrm{F} 1$ and $\mathrm{R} 1$ primers amplified a $337 \mathrm{bp}$ product of the targeted allele, and F1 and R2 primers amplified a 239 bp product of the WT allele. Absence of Bmyc mRNA from homozygotes was confirmed by RT-PCR using RNA extracted from the adult initial segment epididymis using the F1 and R2 primer pair.

\section{Animal handling}

All animal handling was conducted in accordance with Finnish Animal Ethics Committee and the institutional animal care policies of the University of Turku (Turku, Finland), which fully meet the requirements as defined in the NIH Guide on animal experimentation. Male KO and age-matched WT controls from mixed C57BL/6N-SV129 background were used throughout the study, except where otherwise noted. The animals were housed under controlled environmental conditions $(12 \mathrm{~h}$ light:12 $\mathrm{h}$ darkness; temperature $21 \pm 1{ }^{\circ} \mathrm{C}$ ) at the Animal
Facility of the University of Turku. Soy-free SDS RM3 (Special Diet Service, Whitman, Essex, UK) and tap water were available ad libitum.

\section{Histological analyses}

The mice were anesthetized by i.p. injection of 400-600 $\mu$ l $2.5 \%$ Avertin (2-2-2 tribromoethanol) and blood was collected by cardiac puncture. At the ages of 3 and 6 months, the mice were weighed, and thereafter, the tissues studied were dissected, weighed, and processed for histology. The testes were fixed in Bouin's solution (Sigma-Aldrich), and all other tissues were fixed in $4 \%$ paraformaldehyde (PFA). For histological analyses, the tissues were sectioned at 3-5 $\mu \mathrm{m}$ thickness and stained with Harris's hematoxylin and eosin (BDH Ltd., Poole, UK). At least five mice per group were analyzed. Testes and epididymides from 3- to 4-week-old WT and $\mathrm{KO}$ male mice were similarly collected.

Epididymal duct diameter was measured from histological sections from epididymal segments 3 and 4 (Abou-Haila \& Fain-Maurel 1984) of 3-month-old WT and KO mice. Diameter was measured from five to ten separate duct cross sections of five mice, and a mean value was calculated for each mouse.

\section{Immunohistochemical stainings}

Apoptotic and proliferating cells in the testis and the epididymis were identified by TUNEL assay and Ki-67 labeling respectively. The tissues were PFA fixed, dehydrated, and sectioned at 3-5 $\mu \mathrm{m}$ thickness, and the tissue sections were deparaffinated and rehydrated in xylene and in a series of lowering concentrations of ethanol. Antigen retrieval was performed by boiling the sections in $10 \mathrm{mM}$ sodium citrate in a pressure cooker. For TUNEL staining, the DNA ends were labeled with biotinylated dUTP nucleotides (30 nM; Roche Applied Science) by the terminal transferase enzyme $(1 \mathrm{U} / \mu \mathrm{l}$; Roche) and detected with TexasRed-conjugated avidin (1:50 dilution in TBS with 1\% BSA; Invitrogen) and visualized under fluorescence microscope. Ki-67 staining was performed for similarly treated tissue sections with a goat polyclonal antiKi-67 antibody (1:1000; Santa Cruz Biotechnology, Inc., Santa Cruz, CA, USA). The primary antibody was detected with

Table 1 Primers used in the study.

\begin{tabular}{|c|c|c|c|}
\hline Primer & Sequence & $T_{\mathbf{m}}\left({ }^{\circ} \mathrm{C}\right)$ & Used in \\
\hline \multirow[t]{5}{*}{ Bmyc } & F1: GAGTCAGGCAACTATGGATGAACG & 62.7 & ES cell screening \\
\hline & R1: GGTAGGCAGTTCGCTCCAAGC & 63.7 & ES cell screening \\
\hline & F2: TGGACTATGACTCGGTGCAG & 59.4 & Genotyping \\
\hline & R2: AAAGAACGGAGCCGGTTG & 56.0 & Genotyping \\
\hline & R3: CAGGTCTGCGATGGAGAAGCT & 61.8 & Genotyping \\
\hline \multirow[t]{2}{*}{ Mina } & F1: ACGGCAGAGCACATTTTCTT & 55.3 & qRT-PCR \\
\hline & R1: CCAGCTGCAGGATAAAAACC & 57.3 & qRT-PCR \\
\hline \multirow[t]{2}{*}{ Cdk4 } & F1: TGGTACCGAGCTCCTGAAGT & 59.4 & qRT-PCR \\
\hline & R1: GTCGTCTTCTGGAGGCAATC & 59.4 & qRT-PCR \\
\hline \multirow[t]{2}{*}{ Brca } & F1: AGATGACGAGTCAGGGATGG & 59.4 & qRT-PCR \\
\hline & R1: AGATCCCAGGTATGGGGTTC & 59.4 & qRT-PCR \\
\hline \multirow[t]{2}{*}{ Cdk11b } & F1: TCTGCACATCACCGTACCAT & 57.3 & qRT-PCR \\
\hline & R1: GAACCTGAGCСTGATTCTGC & 59.4 & qRT-PCR \\
\hline \multirow[t]{2}{*}{ Мyc } & F1: TCCTGTACCTCGTCCGATTC & 59.4 & qRT-PCR \\
\hline & R1: GTTGTGCTGGTGAGTGGAGA & 59.4 & qRT-PCR \\
\hline
\end{tabular}


a HRP-linked anti-goat secondary antibody (1:2000; GE Healthcare, Little Chalfont, UK) and visualized with a HRP detection kit (Dako, Glostrup, Denmark).

\section{Sperm analyses}

Sperm were collected from 2- to 6-month-old WT and KO mice. Cauda epididymis was dissected out and incubated in $400 \mu \mathrm{l}$ Dulbecco's PBS at $37^{\circ} \mathrm{C}$ for $15 \mathrm{~min}$. Sperm were spread on object slides, air dried, and fixed for $1 \mathrm{~h}$ in a mixture of ethanol and acetic acid (2:1). After fixation, the slides were air dried, and sperm were stained with Harris' hematoxylin and eosin (BDH Ltd.). A series of dilutions was made from caudal sperm in Dulbecco's PBS, and the sperm were counted in a Bürker haemocytometer chamber (Hawksley, Lancing, Sussex, UK).

\section{Hormone measurements}

Hormone measurements were carried out from serum samples collected from 3-month-old male WT and KO mice. Testosterone was measured from diethyl ether extracts using RIA (Huhtaniemi et al. 1985), and FSH and LH were measured by immunofluorometric assays as described previously (Haavisto et al. 1993, van Casteren et al. 2000). Intratesticular testosterone was analyzed by homogenizing one weighed testis in $5 \mathrm{ml}$ PBS from $11 \mathrm{KO}$ and eight WT mice. The homogenates were diethyl extracted and measured by RIA (Spectric Kit, Orion Diagnostica, Finland).

\section{RNA isolation, RT-PCR, and qRT-PCR}

For RT-PCR, total RNA from testis and epididymides of 1-, 2-, 3-, 4-, and 8-week-old mice was isolated using TRIzol reagent (Invitrogen). RNA $(1 \mu \mathrm{g})$ was treated with DNase (DNase I Amplification Grade Kit, Invitrogen) and a single-step RT-PCR was performed with AMV reverse transcriptase (Promega), RNasin Ribonuclease Inhibitor (Promega), and DyNAzyme II DNA polymerase (FinnZymes). From each sample, a minus-RT control was made by omitting AMV-RT from the reaction mix. Actb expression was determined as a control for RNA stability.

\section{Induction of cell proliferation}

Using a transgene consisting of a GPX5 promoter-driven large T-antigen, we have previously generated two transgenic mouse lines: one with high expression and epididymal tumors (GPX5-Tag1) and the other one with lower level of T-antigen expression leading to epididymal dysfunction and infertility, but no tumor formation (Sipilä et al. 2004). In order to test the role of BMYC in cell proliferation and its potential role as tumor suppressor in the epididymis, the Bmyc KO mice were crossbred with the GPX5-Tag2 transgenic mice (Sipilä et al. 2004). The epididymides were collected from 2- to 12-month-old Bmyc ${ }^{-/-}$; GPX5-Tag2 ${ }^{+/ W T}$ mice, and $\mathrm{BmyC}^{+/+}$; GPX5-Tag2 ${ }^{+\mathrm{NT}}$ mice were used as controls. Morphological and histological comparisons were performed as described earlier.

\section{Statistical analyses}

All statistical comparisons were performed using the SigmaPlot program (Systat Software, Inc., Point Richmond, CA, USA). All pairwise comparisons between WT and $\mathrm{KO}$ values were analyzed by $t$-test, except where otherwise noted. All error bars in figures indicate s.D.

\section{Declaration of interest}

The authors declare that there in no conflict of interest that could be perceived as prejudicing the impartiality of the research reported.

\section{Funding}

This work was supported by grants from The Academy of Finland (project numbers 211480 and 121527) and Sigrid Jusélius Foundation.

\section{Acknowledgements}

Bmyc $\mathrm{KO}$ mice were generated in collaboration with Turku Center for Disease Modeling, TCDM, Turku, Finland, (www. tcdm.fi), and the authors thank the TCDM personnel for skillful assistance in various stages of this study. They also thank $\mathrm{T}$ Leinonen for technical assistance with the histology specimens and $\mathrm{N}$ Kotaja for help in analyzing testicular samples.

\section{References}

Abou-Haila A \& Fain-Maurel MA 1984 Regional differences of the proximal part of mouse epididymis: morphological and histochemical characterization. Anatomical Record 209 197-208. (doi:10.1002/ar.1092090207)

Albihn A, Johnsen JI \& Henriksson MA 2010 MYC in oncogenesis and as a target for cancer therapies. Advances in Cancer Research 107 163-224. (doi:10.1016/S0065-230X(10)07006-5)

Asker CE, Magnusson KP, Piccoli SP, Andersson K, Klein G, Cole MD \& Wiman KG 1995 Mouse and rat B-myc share amino acid sequence homology with the c-myc transcriptional activator domain and contain a B-myc specific carboxy terminal region. Oncogene 11 1963-1969.

Blackwood EM \& Eisenman RN 1991 Max: a helix-loop-helix zipper protein that forms a sequence-specific DNA-binding complex with Myc. Science 251 1211-1217. (doi:10.1126/science.2006410)

Buchold GM, Magyar PL \& O'Brien DA 2007 Mice lacking cyclindependent kinase inhibitor p19Ink4d show strain-specific effects on male reproduction. Molecular Reproduction and Development 74 1008-1020. (doi:10.1002/mrd.20715)

Burton RA, Mattila S, Taparowsky EJ \& Post CB 2006 B-myc: N-terminal recognition of myc binding proteins. Biochemistry 45 9857-9865. (doi:10.1021/bi060379n)

van Casteren JI, Schoonen WG \& Kloosterboer HJ 2000 Development of time-resolved immunofluorometric assays for rat follicle-stimulating hormone and luteinizing hormone and application on sera of cycling rats. Biology of Reproduction 62 886-894. (doi:10.1095/biolreprod62. 4.886)

Cole MD \& McMahon SB 1999 The Myc oncoprotein: a critical evaluation of transactivation and target gene regulation. Oncogene 18 2916-2924. (doi:10.1038/sj.onc.1202748)

Cornwall GA, Collis R, Xiao Q, Hsia N \& Hann SR 2001 B-Myc, a proximal caput epididymal protein, is dependent on androgens and testicular factors for expression. Biology of Reproduction 64 1600-1607. (doi:10.1095/biolreprod64.6.1600) 
Davis AC, Wims M, Spotts GD, Hann SR \& Bradley A 1993 A null c-myc mutation causes lethality before 10.5 days of gestation in homozygotes and reduced fertility in heterozygous female mice. Genes and Development 7 671-682. (doi:10.1101/gad.7.4.671)

Domashenko AD, Latham KE \& Hatton KS 1997 Expression of myc-family, myc-interacting, and myc-target genes during preimplantation mouse development. Molecular Reproduction and Development 47 57-65. (doi:10.1002/(SICI)1098-2795(199705)47:1 < 57::AID-MRD8 > 3.0.CO;2-P)

Ganem JP, Jhaveri FM \& Marroum MC 1998 Primary adenocarcinoma of the epididymis: case report and review of the literature. Urology 52 904-908. (doi:10.1016/S0090-4295(98)00275-1)

Gregory MA, Xiao Q, Cornwall GA, Lutterbach B \& Hann SR 2000 B-Myc is preferentially expressed in hormonally-controlled tissues and inhibits cellular proliferation. Oncogene 19 4886-4895. (doi:10.1038/sj.onc. 1203851)

Haavisto AM, Pettersson K, Bergendahl M, Perheentupa A, Roser JF \& Huhtaniemi I 1993 A supersensitive immunofluorometric assay for rat luteinizing hormone. Endocrinology 132 1687-1691. (doi:10.1210/en. 132.4.1687)

Henriksson M \& Luscher B 1996 Proteins of the Myc network: essential regulators of cell growth and differentiation. Advances in Cancer Research 68 109-182.

Hoffman B \& Liebermann DA 2008 Apoptotic signaling by c-MYC. Oncogene 27 6462-6472. (doi:10.1038/onc.2008.312)

Huhtaniemi I, Nikula H \& Rannikko S 1985 Treatment of prostatic cancer with a gonadotropin-releasing hormone agonist analog: acute and long term effects on endocrine functions of testis tissue. Journal of Clinical Endocrinology and Metabolism 61 698-704. (doi:10.1210/jcem-61-4-698)

Hurlin PJ $2005 \mathrm{~N}-M y c$ functions in transcription and development. Birth Defects Research. Part C, Embryo Today: Reviews 75 340-352. (doi:10.1002/bdrc.20059)

Ingvarsson S, Asker C, Axelson H, Klein G \& Sumegi J 1988 Structure and expression of B-myc, a new member of the myc gene family. Molecular and Cellular Biology 8 3168-3174. (doi:10.1128/MCB.8.8.3168)

Kodaira K, Takahashi R, Hirabayashi M, Suzuki T, Obinata M \& Ueda M 1996 Overexpression of c-myc induces apoptosis at the prophase of meiosis of rat primary spermatocytes. Molecular Reproduction and Development 45 403-410. (doi:10.1002/(SICI)1098-2795(199612)45:4 $<403:: A I D-M R D 1>3.0 . C O ; 2-V)$

McDermott J, Zhao Y \& Sauer B 2004 A simple polymerase chain reaction screen for homologous targeting in embryonic stem cells. Analytical Biochemistry 332 401-403. (doi:10.1016/j.ab.2004.05.027)
Menssen A \& Hermeking H 2002 Characterization of the c-MYC-regulated transcriptome by SAGE: identification and analysis of c-MYC target genes. PNAS 99 6274-6279. (doi:10.1073/pnas.082005599)

Muyrers JP, Zhang Y, Testa G \& Stewart AF 1999 Rapid modification of bacterial artificial chromosomes by ET-recombination. Nucleic Acids Research 27 1555-1557. (doi:10.1093/nar/27.6.1555)

Resar LM, Dolde C, Barrett JF \& Dang CV 1993 B-myc inhibits neoplastic transformation and transcriptional activation by c-myc. Molecular and Cellular Biology 13 1130-1136. (doi:10.1128/MCB.13.2.1130)

Salacz ME, Watson KR \& Schomas DA 2011 Glioblastoma: Part I. Current state of affairs. Missouri Medicine 108 187-194.

Sipilä P, Shariatmadari R, Huhtaniemi IT \& Poutanen M 2004 Immortalization of epididymal epithelium in transgenic mice expressing simian virus 40 T antigen: characterization of cell lines and regulation of the polyoma enhancer activator 3. Endocrinology 145 437-446. (doi:10.1210/en. 2003-0831)

Sipilä P, Jalkanen J, Huhtaniemi IT \& Poutanen M 2009 Novel epididymal proteins as targets for the development of post-testicular male contraception. Reproduction 137 379-389. (doi:10.1530/REP-08-0132)

Suzuki M, Abe K, Yoshinaga K, Obinata M, Furusawa M \& Abe K 1996 Specific arrest of spermatogenesis caused by apoptotic cell death in transgenic mice. Genes to Cells 1 1077-1086. (doi:10.1046/j.13652443.1996.d01-228.x)

Tsuneoka M, Nishimune Y, Ohta K, Teye K, Tanaka H, Soejima M, lida H, Inokuchi T, Kimura H \& Koda Y 2006 Expression of Mina53, a product of a Myc target gene in mouse testis. International Journal of Andrology 29 323-330. (doi:10.1111/j.1365-2605.2005.00572.x)

Zhang Y, Buchholz F, Muyrers JP \& Stewart AF 1998 A new logic for DNA engineering using recombination in Escherichia coli. Nature Genetics 20 123-128. (doi:10.1038/2417)

Zhang Y, Muyrers JP, Testa G \& Stewart AF 2000 DNA cloning by homologous recombination in Escherichia coli. Nature Biotechnology 18 1314-1317. (doi:10.1038/78475)

Received 16 November 2011

First decision 7 December 2011

Revised manuscript received 6 July 2012

Accepted 6 August 2012 